\title{
IAMJ
}

INTERNATIONAL

AYURVEDIC

MEDICAL JOURNAL

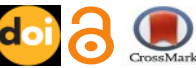

Research Article

ISSN: 23205091

Impact Factor: 5.344

\section{A COMPARATIVE CLINICAL STUDY ON THE EFFICACY OF MARMA CHIKITSA AND STIMULATION OF MARMA POINTS USING TENS IN MANAGEMENT OF CERVICAL SPONDYLOSIS}

\section{Rajkiran$^{1}$, D. Gururaja ${ }^{2}$}

${ }^{1}$ Final year P.G Scholar, Department of Shalya Tantra, MIAMS, Manipal - 576104, Karnataka, India

${ }^{2}$ M.S. (Ay.), PhD (Japan), Professor and Head, Department of Post Graduate Studies in Shalya Tantra, MIAMS, Manipal - 576104, Karnataka, India

Corresponding Author:1rajkiran4444@gmail.com

\section{https://doi.org/10.46607/iamj07p4052020}

(Published online: July 2020)

Open Access

(C) International Ayurvedic Medical Journal, India 2020

Article Received: 20/08/2020 - Peer Reviewed: 23/08/2020 - Accepted for Publication: 23/08/2020

Check for updates

\section{ABSTRACT}

Background: Cervical Spondylosis a degenerative disc disease, pain being the main first concern along with stiffness and decreased movements of neck. Marma chikitsa is done by employing electrical stimulation using TENS or adapting Varma chikitsa explained in Siddha system of medicine on Marma points explained by Acharya Sushruta.

Objectives: To compare the efficacy of Marma manipulation and electrical stimulation using TENS in the management of Cervical Spondylosis.

Methods: 40 Subjects diagnosed with Cervical Spondylosis was divided into 2 groups of 20 each. Group A was treated with TENS and Group B was treated with Marma Chikitsa for 7 days respectively.

Results: The outcome of treatment after 14 days was statistically significant based in criteria taken for study.

Interpretation \& Conclusion: Group A showed better effect in reducing stiffness, pain, and improves angle of flexion, extension, side bending, tendon reflexes, power of hand muscle and rotation of neck. Whereas Group B was effective in improving the restricted movements, tenderness and mild improvement on power of hand muscles. 
Keywords: Marma Chikitsa, TENS, Cervical Spondylosis

\section{INTRODUCTION}

Neck pain is a common musculoskeletal problem which affects a substantial proportion of people in there later lives. It has long been recognized that neck pain can result in symptoms and problems being referred into the upper limb.

Cervical Spondylosis is a degenerative condition that effects the cervical spine. Pain and stiffness are the primary symptoms. Often, there are referred symptoms in the upper limb. Radiation of pain occurs from shoulder to digits along the course of the nerve indicates nerve root compression ${ }^{1}$.

Ayurvedic literature explains that Vata Dosha is responsible for all sorts of movements of the body and its derangements leads to loss of the same. Vishwachi, Manyasthambha, Greevastambha and Avabahuka disorders comes Under Urdhwa jatrugatavikaras and 80 Nanatmaja Vikaras of Vata as separate entity ${ }^{2,3}$ with their signs and symptoms ${ }^{4}$, which are having certain similarities with cervical spondylosis, Ayurveda explains many vital points of anatomical and physiological significance. These points are called as "Marma"5 Prana circulates throughout the body and these Marma points acts as junctions where Prana is specially seated. Any injury affecting the Marma Sthana results in disturbance in the flow of Prana leading to the manifestation of the disease. Manipulating and stimulating Marmas in proper manner improves flow of Prana in the body. In Ayurveda they explained Marma points but there is lack of description about Marma Chikitsa. Siddha system $^{6}$ of medicine gives detailed description about Marma Chikitsa and manipulation methods over these points.

Electrical stimulation of acupuncture points in the management of pain and symptoms have been explained in Acupuncture ${ }^{7}$ of Traditional Chinese system of medicines

So proper knowledge of Marma along with manipulation and electrical stimulation may show better results. No study has been carried out on this, the study is planned to evaluate and to compare the efficacy of Marma manipulation and electrical stimulation using
Transcutaneous Electrical Nerve Stimulation (TENS) on Marma points in the management of Cervical spondylosis.

\section{Objectives}

- To study the effect of manipulation of Marma points as in the management of Cervical Spondylosis.

- To study the effect of electrical stimulation on Marma points using TENS in the management of Cervical Spondylosis.

- To compare the efficacy of Marma manipulation and electrical stimulation using TENS in the management of Cervical Spondylosis.

\section{Materials and Methods}

\section{Sources of data}

\section{Clinical source:}

40 Patients attending the OPD and IPD of Muniyal Institute of Ayurveda medical science were selected for the study with their consent.

\section{Method of collection of data}

A detailed case Proforma was prepared which included the detailed history, physical signs and symptoms of Cervical spondylosis.

\section{Inclusion Criteria:}

Clinically diagnosed cases of Cervical spondylosis were taken for the study.

- Subjects of age group- 20 to 70 years (Irrespective of gender).

- Patients fit for Marma Chikitsa

- Patients presenting with the signs and symptoms of Cervical Spondylosis.

\section{Exclusion Criteria:}

- Patient contraindicated for Marma Chikitsa

- Patient with traumatic injury of Cervical Spine

- Patient with major systemic disorders neurodegenerative condition of spine like tb malignancies etc. that may interfere with the course of treatment.

\section{Laboratory Investigations:}

- Complete blood test

- Plain X-ray of Cervical spine (AP and LAT)

- MRI (if necessary) 


\section{Interventions:}

Marma Points located in neck and upper extremity involved in Cervical spondylosis was selected and Manipulation/TENS were given.

\section{Group-A}

Procedure $\quad-\quad$ Stimulation using TENS

Time $\quad$ - $\quad 20$ minutes

\begin{tabular}{|c|c|}
\hline Duration of treatment - & 7 days \\
\hline Follow up & on $14^{\text {th }}$ day \\
\hline \multicolumn{2}{|l|}{ Group-B } \\
\hline Procedure & Marma chikitsa \\
\hline Time & 20 minutes \\
\hline Duration of treatment - & 7 days \\
\hline Follow up & on $14^{\text {th }}$ day \\
\hline
\end{tabular}

\section{Table 1}

\begin{tabular}{|c|c|c|c|c|}
\hline S.NO. & Marma & Varma $^{8}$ & Manipulation technique & Stimulation technique TENS ${ }^{9}$ \\
\hline 1. & Krikataka & Porchaikalam & $\begin{array}{l}\text { Using } 1 / 2 \text { Maathirai pressure, apply clock- } \\
\text { wise rotation } 3 \text { times and gently drag up } \\
\text { to the tip of right shoulder similarly anti- } \\
\text { clockwise rotation } 3 \text { times and drag to the } \\
\text { tip of the left shoulder. }\end{array}$ & \multirow{9}{*}{$\begin{array}{l}\text { A continuous electrical wave of } \\
\text { intensity (amplitude), high fre- } \\
\text { quency (10-200 pps) for } 20 \\
\text { minutes. }\end{array}$} \\
\hline 2. & Amsa & Mudichu & $\begin{array}{l}\text { Using } 1 / 4 \text { Maathirai pressure, press and re- } \\
\text { lease } 3 \text { times }\end{array}$ & \\
\hline 3. & Amsaphalaka & Kaichulukki & $\begin{array}{l}\text { Using } 1 / 4 \text { Maathirai pressure, press and re- } \\
\text { lease } 3 \text { times }\end{array}$ & \\
\hline 4. & Brihati & Chhipi & $\begin{array}{l}\text { Using } 1 / 4 \text { Maathirai pressure, apply up- } \\
\text { ward and downward movement } 3 \text { times }\end{array}$ & \\
\hline 5. & Kakshadhara & Chavvu & $\begin{array}{l}\text { Press and release Kshipra and Lohitaksha } \\
\text { marma alternately for } 3 \text { times. }\end{array}$ & \\
\hline 6. & Kurpara & Koimottu & $\begin{array}{l}\text { Press and release } 3 \text { times by using } 1 / 4 \\
\text { Maathirai pressure. }\end{array}$ & \\
\hline 7. & Indrabasti & Teetha & $\begin{array}{l}\text { Press and release } 3 \text { times by using } 1 / 4 \\
\text { Maathirai pressure. }\end{array}$ & \\
\hline 8. & Manibandha & Manibandha & $\begin{array}{l}\text { Press and release } 3 \text { times by using } 1 / 4 \\
\text { Maathirai pressure. }\end{array}$ & \\
\hline 9. & Kshipra & Kawlikalam & $\begin{array}{l}\text { Press and release } 3 \text { times by using pulp } \\
\text { part of middle three fingers. }\end{array}$ & \\
\hline
\end{tabular}

Maathirai - pressure exerted by the pulp of the thumb.

During TENS Fig: 2, pulsed electrical currents are generated by a small battery-operated TENS device through which Currents from the TENS device was delivered through the skin by two self-adhering electrode pads.

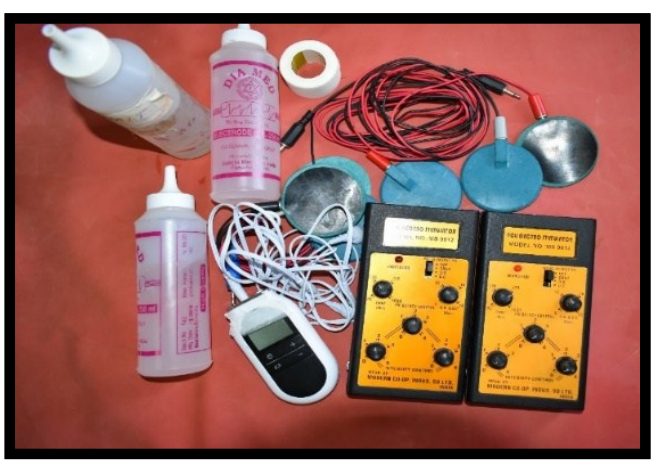

Fig: 1

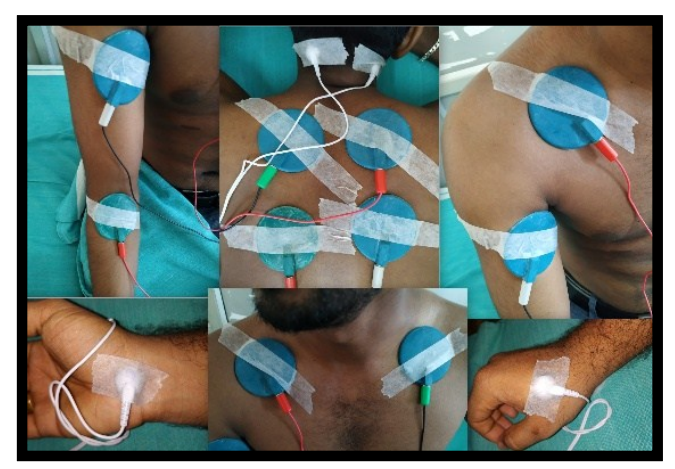

Fig: 2 


\section{Assessment Criteria}

Subjective

- Visual analogue scale (for pain assessment)

- Stiffness

\section{Objective}

- Tenderness

- Angle of neck movement.

- Neck Disability Index, NDI. ${ }^{10}$

Measured by using Goniometer and based on degree obtained by using Goniometer, statistically values were analysed.

\section{Observations and Results}

Table 2

\begin{tabular}{|c|c|c|c|c|c|c|c|c|c|}
\hline \multirow[t]{2}{*}{ Group A } & \multirow[t]{2}{*}{ BT Mean } & & & \multirow[t]{2}{*}{ DIFF } & \multirow[t]{2}{*}{$\%$} & \multicolumn{4}{|c|}{ Wilcoxon rank test } \\
\hline & & & & & & SD & SEM & $\mathrm{P}$ & Significant \\
\hline \multirow[t]{2}{*}{ Pain } & \multirow[t]{2}{*}{5.85} & AT & 3.200 & 2.65 & 45.3 & 0.812 & 0.181 & $<0.001$ & $\mathrm{ES}$ \\
\hline & & $\mathrm{AF}$ & 2.850 & 3.00 & 52.3 & 1.170 & 0.261 & $<0.001$ & ES \\
\hline \multirow[t]{2}{*}{ Stiffness } & \multirow[t]{2}{*}{2.45} & AT & 1.500 & 0.95 & 38.8 & 0.510 & 0.114 & $<0.001$ & ES \\
\hline & & $\mathrm{AF}$ & 1.200 & 1.25 & 51.0 & 0.638 & 0.143 & $<0.001$ & ES \\
\hline \multirow[t]{2}{*}{ Restricted Movements } & \multirow[t]{2}{*}{1.95} & AT & 1.400 & 0.55 & 28.2 & 0.510 & 0.114 & $<0.001$ & ES \\
\hline & & $\mathrm{AF}$ & 1.350 & 0.60 & 30.8 & 0.502 & 0.112 & $<0.001$ & $\mathrm{ES}$ \\
\hline \multirow[t]{2}{*}{ Flexion } & \multirow[t]{2}{*}{31.5} & AT & 39.00 & -7.50 & 23.8 & 2.565 & 0.573 & $<0.001$ & $\mathrm{ES}$ \\
\hline & & $\mathrm{AF}$ & 38.50 & -7.00 & 22.3 & 4.413 & 0.986 & $<0.001$ & $\mathrm{ES}$ \\
\hline \multirow[t]{2}{*}{ Extension } & \multirow[t]{2}{*}{30.25} & AT & 37.25 & -7.25 & 24.0 & 2.552 & 0.570 & $<0.001$ & ES \\
\hline & & $\mathrm{AF}$ & 36.00 & -5.75 & 19.0 & 4.375 & 0.978 & $<0.001$ & ES \\
\hline \multirow[t]{2}{*}{ Rotation } & \multirow[t]{2}{*}{36.75} & AT & 44.00 & -7.25 & 19.8 & 3.024 & 0.676 & $<0.001$ & ES \\
\hline & & $\mathrm{AF}$ & 46.75 & -10.0 & 27.3 & 3.627 & 0.811 & $<0.001$ & ES \\
\hline \multirow[t]{2}{*}{ Side Bending } & \multirow[t]{2}{*}{29.00} & AT & 35.25 & -6.25 & 21.5 & 3.582 & 0.801 & $<0.001$ & ES \\
\hline & & $\mathrm{AF}$ & 36.00 & -7.00 & 24.2 & 3.770 & 0.843 & $<0.001$ & ES \\
\hline \multirow[t]{2}{*}{ Tenderness } & \multirow[t]{2}{*}{2.10} & AT & 1.350 & 0.75 & 35.7 & 0.444 & 0.099 & $<0.001$ & ES \\
\hline & & $\mathrm{AF}$ & 0.900 & 1.20 & 57.1 & 0.410 & 0.091 & $<0.001$ & ES \\
\hline \multirow[t]{2}{*}{ Power of Hand muscles } & \multirow[t]{2}{*}{3.90} & AT & 4.45 & -0.55 & 14.1 & 0.510 & 0.114 & $<0.001$ & ES \\
\hline & & $\mathrm{AF}$ & 4.55 & -0.65 & 16.7 & 0.489 & 0.109 & $<0.001$ & ES \\
\hline \multirow[t]{2}{*}{ Tendon reflexes } & \multirow[t]{2}{*}{2.85} & AT & 3.60 & -0.75 & 26.4 & 0.550 & 0.123 & $<0.001$ & ES \\
\hline & & $\mathrm{AF}$ & 3.85 & -1.00 & 35.1 & 0.458 & 0.102 & $<0.001$ & ES \\
\hline \multirow[t]{2}{*}{ Neck Disability Index } & \multirow[t]{2}{*}{12.4} & AT & 8.45 & 3.95 & 31.9 & 0.825 & 0.184 & $<0.001$ & ES \\
\hline & & $\mathrm{AF}$ & 5.35 & 7.05 & 56.9 & 1.317 & 0.294 & $<0.001$ & ES \\
\hline
\end{tabular}

Statistical analysis: The scores of assessment criteria were analyzed statistically in the form of mean score B.T (Before Treatment), A.T. (after Treatment), Difference of mean (B.T. - A.T), S.D. (Standard Deviation), S.E (Standard Error). The Wilcoxon Signed-Ranks Test was carried out for within the groups and Mann Whitney $U$ test for between the groups using GraphPad InStat and SigmaStat 4.0. The results were considered Significant or Insignificant depending upon P value.

Table 3

\begin{tabular}{|c|c|c|c|c|c|c|c|c|c|}
\hline \multirow{2}{*}{ Group B } & \multirow[t]{2}{*}{ BT Mean } & & & \multirow[t]{2}{*}{ DIFF } & \multirow[t]{2}{*}{$\%$} & \multicolumn{4}{|c|}{ Wilcoxon rank test } \\
\hline & & & & & & SD & SEM & $\mathrm{P}$ & Significant \\
\hline \multirow[t]{2}{*}{ Pain } & \multirow[t]{2}{*}{6.6} & AT & 4.65 & 1.95 & 28.0 & 1.099 & 0.245 & $<0.001$ & ES \\
\hline & & $\mathrm{AF}$ & 4.50 & 2.1 & 31.9 & 0.788 & 0.176 & $<0.001$ & ES \\
\hline \multirow{2}{*}{ Stiffness } & \multirow[t]{2}{*}{2.15} & AT & 1.55 & 0.60 & 28.0 & 0.502 & 0.112 & $<0.001$ & ES \\
\hline & & $\mathrm{AF}$ & 1.50 & 0.65 & 30.2 & 0.489 & 0.109 & $<0.001$ & ES \\
\hline
\end{tabular}




\begin{tabular}{|c|c|c|c|c|c|c|c|c|c|}
\hline \multirow[t]{2}{*}{ Restricted Movements } & \multirow[t]{2}{*}{1.85} & AT & 1.50 & 0.35 & 19 & 0.489 & 0.109 & 0.0156 & $\mathrm{~S}$ \\
\hline & & $\mathrm{AF}$ & 1.25 & 0.60 & 32.5 & 0.598 & 0.133 & 0.0020 & ES \\
\hline \multirow[t]{2}{*}{ Flexion } & \multirow[t]{2}{*}{31.0} & AT & 34.0 & -3.00 & 9.7 & 2.513 & 0.562 & $<0.001$ & ES \\
\hline & & $\mathrm{AF}$ & 33.5 & -2.50 & 8.2 & 3.441 & 0.769 & 0.0166 & $\mathrm{~S}$ \\
\hline \multirow[t]{2}{*}{ Extension } & \multirow[t]{2}{*}{29.5} & AT & 33.5 & -4.00 & 13.6 & 3.078 & 0.688 & $<0.001$ & ES \\
\hline & & $\mathrm{AF}$ & 34.0 & -4.50 & 15.3 & 4.560 & 1.020 & $<0.001$ & ES \\
\hline \multirow[t]{2}{*}{ Rotation } & \multirow[t]{2}{*}{37.5} & AT & 41.25 & -3.75 & 10.1 & 2.221 & 0.496 & $<0.001$ & ES \\
\hline & & $\mathrm{AF}$ & 42.25 & -4.75 & 12.8 & 4.435 & 0.991 & $<0.001$ & ES \\
\hline \multirow[t]{2}{*}{ Side Bending } & \multirow[t]{2}{*}{30.5} & AT & 34.0 & -3.50 & 11.5 & 3.285 & 0.734 & $<0.001$ & ES \\
\hline & & $\mathrm{AF}$ & 35.0 & -4.50 & 12.9 & 3.940 & 0.881 & $<0.001$ & ES \\
\hline \multirow[t]{2}{*}{ Tenderness } & \multirow[t]{2}{*}{1.8} & AT & 1.40 & 0.40 & 22.2 & 0.502 & 0.112 & 0.0080 & VS \\
\hline & & $\mathrm{AF}$ & 1.05 & 0.75 & 41.6 & 0.444 & 0.099 & $<0.001$ & ES \\
\hline \multirow[t]{2}{*}{ Power of Hand muscles } & \multirow[t]{2}{*}{3.85} & AT & 4.30 & -0.45 & 11.7 & 0.510 & 0.114 & 0.0040 & VS \\
\hline & & $\mathrm{AF}$ & 4.35 & -0.50 & 13.0 & 0.513 & 0.114 & 0.0020 & VS \\
\hline \multirow[t]{2}{*}{ Tendon reflexes } & \multirow[t]{2}{*}{2.80} & AT & 3.25 & -0.45 & 16.1 & 0.604 & 0.135 & 0.0140 & $\mathrm{~S}$ \\
\hline & & $\mathrm{AF}$ & 3.60 & -0.80 & 28.6 & 0.523 & 0.117 & $<0.001$ & ES \\
\hline \multirow[t]{2}{*}{ Neck Disability Index } & \multirow[t]{2}{*}{12.5} & AT & 9.65 & 2.85 & 22.8 & 0.670 & 0.150 & $<0.001$ & ES \\
\hline & & $\mathrm{AF}$ & 7.40 & 5.10 & 40.8 & 1.021 & 0.228 & $<0.001$ & ES \\
\hline
\end{tabular}

Table 4:

\begin{tabular}{|c|c|c|c|c|c|c|c|c|c|}
\hline \multirow[t]{2}{*}{ PARAMETER } & \multicolumn{2}{|c|}{ MEAN } & \multirow{2}{*}{$\begin{array}{c}\text { Mean } \\
\text { diff }\end{array}$} & \multicolumn{2}{|c|}{ SD } & \multicolumn{2}{|c|}{ SE } & \multirow{2}{*}{$\begin{array}{c}\mathrm{P} \\
\text { value }\end{array}$} & \multirow[t]{2}{*}{ Result } \\
\hline & $\begin{array}{c}\text { Group } \\
\text { A }\end{array}$ & $\begin{array}{l}\text { Group } \\
\text { B }\end{array}$ & & $\begin{array}{c}\text { Group } \\
\text { A }\end{array}$ & $\begin{array}{c}\text { Group } \\
\text { B }\end{array}$ & $\begin{array}{c}\text { Group } \\
\text { A }\end{array}$ & $\begin{array}{c}\text { Group } \\
\text { B }\end{array}$ & & \\
\hline \multirow[t]{2}{*}{ Pain } & 3.200 & 4.65 & -1.45 & 1.005 & 1.268 & 0.224 & 0.283 & $<0.001$ & ES \\
\hline & 2.850 & 4.50 & -1.65 & 0.745 & 0.827 & 0.166 & 0.185 & $<0.001$ & ES \\
\hline \multirow[t]{2}{*}{ Stiffness } & 1.500 & 1.55 & -0.05 & 0.760 & 0.604 & 0.170 & 0.135 & 0.805 & NS \\
\hline & 1.200 & 1.50 & -0.30 & 0.615 & 0.888 & 0.137 & 0.198 & 0.313 & NS \\
\hline \multirow{2}{*}{$\begin{array}{l}\text { Restricted Move- } \\
\text { ments }\end{array}$} & 1.400 & 1.50 & -0.10 & 0.680 & 0.513 & 0.152 & 0.114 & 0.793 & NS \\
\hline & 1.350 & 1.25 & -0.10 & 0.933 & 0.786 & 0.208 & 0.175 & 0.733 & NS \\
\hline \multirow[t]{2}{*}{ Flexion } & 39.00 & 34.0 & 5.00 & 4.168 & 4.757 & 0.931 & 1.064 & 0.002 & VS \\
\hline & 38.50 & 33.5 & 5.00 & 5.643 & 3.663 & 1.262 & 0.819 & 0.006 & $\mathrm{~S}$ \\
\hline \multirow[t]{2}{*}{ Extension } & 37.50 & 33.5 & 4.00 & 4.443 & 3.663 & 0.993 & 0.819 & 0.0085 & NS \\
\hline & 36.00 & 34.0 & 2.00 & 5.026 & 3.078 & 1.124 & 0.688 & 0.1295 & NS \\
\hline \multirow[t]{2}{*}{ Rotation } & 44.00 & 41.25 & 2.75 & 4.757 & 5.350 & 1.064 & 1.196 & 0.152 & NS \\
\hline & 46.75 & 42.25 & 4.50 & 5.447 & 5.955 & 1.218 & 1.332 & 0.123 & NS \\
\hline \multirow[t]{2}{*}{ Side Bending } & 35.25 & 34.0 & 1.25 & 3.796 & 3.839 & 0.848 & 1.124 & 0.354 & NS \\
\hline & 36.00 & 35.0 & 1.00 & 5.026 & 3.627 & 0.858 & 0.811 & 0.401 & NS \\
\hline \multirow[t]{2}{*}{ Tenderness } & 1.350 & 1.40 & -0.05 & 0.489 & 0.598 & 0.109 & 0.133 & 0.933 & NS \\
\hline & 0.900 & 1.05 & -0.15 & 0.552 & 0.604 & 0.123 & 0.135 & 0.498 & NS \\
\hline \multirow{2}{*}{$\begin{array}{l}\text { Power of Hand } \\
\text { Muscles }\end{array}$} & 4.450 & 4.30 & 0.15 & 0.510 & 0.470 & 0.114 & 0.105 & 0.414 & NS \\
\hline & 4.550 & 4.35 & 0.20 & 0.510 & 0.489 & 0.114 & 0.109 & 0.276 & NS \\
\hline \multirow[t]{2}{*}{ Tendon Reflexes } & 3.60 & 3.25 & 0.35 & 0.598 & 0.638 & 0.133 & 0.142 & 0.100 & NS \\
\hline & 3.85 & 3.60 & 0.25 & 0.366 & 0.502 & 0.081 & 0.112 & 0.170 & NS \\
\hline \multirow{2}{*}{$\begin{array}{l}\text { Neck Disability In- } \\
\text { dex }\end{array}$} & 8.45 & 9.65 & -1.20 & 1.669 & 2.033 & 0.373 & 0.454 & 0.0635 & NS \\
\hline & 5.35 & 7.40 & -2.05 & 1.461 & 2.257 & 0.326 & 0.504 & 0.0047 & $\mathrm{~S}$ \\
\hline
\end{tabular}

*BT-Before treatment, *AT-After treatment, *AF-After follow up* DIFF-Difference, *SD-Standard deviation, *SEM-Standard error of mean, *ES-Extremely significant, *VS-Very significant, *S-Significant, *NS-Nothing significant. 


\section{DISCUSSION}

\section{Comparison of Marma and Varma points}

The anatomical location and functioning of the marma and Varma are almost similar. These are the vital points of the body where prana or the vital energy is situated. In Ayurveda Sushruta used Marma points to understand Abhigata Lakshanas, but in siddha system of medicine these points are used for diagnosis and therapeutic purpose similarly the study involves comparing it with TENS on these Marma points.

Group A (TENS) showed better effect in reducing stiffness, pain, and improves angle of flexion, extension, side bending, tendon reflexes, power of hand muscle, rotation of neck and NDI. whereas Group B (Marma Chikitsa) was effective in improving the restricted movements, tenderness and mild improvement on power of hand muscles.

Probable mode of action of Marma chikitsa: As in Cervical Spondylosis there is compression of the nerve fibres of the Cervical spine and all the treatment modalities are not so effective as they provide pain relief for some time. Marma Chikitsa which is the precise art of touching an individual in exactly the right place at a critical moment in time, for the purpose of healing and serve as point of access to the body's innate intelligence, opening the doorway to health and wellbeing can lead to release in that energy blockage and can lead to permanent cure .

The Marma chikitsa of stimulation or manipulation can be used as a part of a rejuvenation therapy or it could be used as preventive measure from unwanted conditions. Either way, Marma chikitsa is really useful to help improve or maintain a healthy balance.

\section{Probable mode of action of tens:}

Neurologic effect: The gate control theory of pain by which the electrical stimulation of the large nerve fibres (pressure \& touch) results in impulses that travel faster (because they are myelinated) than do those along the smaller fibres, which transmits the pain.

The electrical stimuli, pressure and touch impulses from TENS arrive faster at the levels of the spinal cord stimulate pain causing nerve endings resulting in a suppression of pain signals. Further it elicits an almost immediate response after application of TENS therapy.
Pharmacologic effect: TENS causes an activation of endogenous analgesic systems involving endorphins, thereby increasing their plasma levels. And gives far more analgesic efficacy than oral analgesics.

Physiologic effect: Mild rhythmic muscle movements will cause an increase in blood and lymph circulation which leads to reduced interstitial edema and accumulation of noxious tissue metabolites, thereby improving the physiological state of muscle which in turn leads to significant reduction in muscle spasm, stiffness and mainly pain.

\section{CONCLUSION}

Marma and Varma are similar, technique of stimulation of Varma points explained in Siddha System of Medicine can be adopted over Marma points of Ayurveda system of medicine. Marma therapy and TENS over Ayurveda Marma points can be effectively implemented for the management of pain, stiffness and tenderness.

\section{REFERENCES}

1. Bhusal Nirmal, Bhated Santosh Kumar. Management of Cervical Spondylosis with Dashamoola Oil Manya Vasti and Internal Medicines-A Case Study, International Journal of Health Sciences \& Research (www.ijhsr.org) Vol.6; Issue: 9; September 2016 page 524.

2. Pandit Sharangadhara acharya son of Pt Damodara, SHARGANDHARA samhita with commentaries Adhamalla's Dipika and Kasirama's Gudhartha Dipika, Chaukhamba Publications, reprint 2013. pp 398 pages no 103.

3. Agnivesha, Charaka Samhita elaborated by Charaka and Drdhabala with The Ayurveda Deepika commentary by Chakrapanidatta on Siddhi sthana edited by Vaidya Yadavji Trikamji Acharya, Chaukamba Surbharathi, Prakashan, Varanasi, Reprint 2008.Pp:738, Pgno:722.

4. Vridha vagbhata, Ashtanga Samgraha edited by Dr Ravidutt Tripathi, Chaukhamba publication, Varanasi, 2003 edition, Sutrasthana 20th chapter sloka no 11 pages no 399.

5. Acharya YT(Ed), Sushruta Samhita of Sushruta with Nibandha Sangraha Commentary by Shri Dalhanacharya and the Nyayachandrika Panjika of Sri 
Gayadasacharya on Nidanasthana Varanasi, Chaukhambha Sanskrit Sansthan, Reprint 2014, pp-824, page-369,372.

6. Shanmugom N, Basic concepts of medical - kaibhagam and seibhagam, Thirumoolar vermology institute, April 2017, $1^{\text {st }}$ edition, Coimbatore, pp217.

7. Agarwal A. L. and Sharma G.N, Advanced Acupuncture Therapy, CBS Publishers and Distributors, $1^{\text {stedi- }}$ tion 2009, reprint 2016, pp-500, pages 73-92.

8. Shanmugom N, Basic concepts of medical varmalogy handbook on varmam therapy -kai bhagam and sei bhagam, thirumoolar vermology institute, 2017, $1^{\text {st }}$ edition, Coimbatore, pp-228 pages 90-154.

9. https://www.ncbi.nlm.nih.gov/pmc/articles/PMC3066716/ last accessed on 15/08/19.

10. Vernon H, Mior S. The neck disability index: A study of reliability and validity. Journal of Manipulative and Physiological Therapeutics, 1991, 14:409-15.

\section{Source of Support: Nil \\ Conflict of Interest: None Declared}

How to cite this URL: Rajkiran \& D. Gururaja: A Comparative Clinical Study On The Efficacy Of Marma Chikitsa And Stimulation Of Marma Points Using Tens In Management Of Cervical Spondylosis. International Ayurvedic Medical Journal \{online\} 2020 \{cited July, 2020\} Available from: http://www.iamj.in/posts/images/upload/2408_2414.pdf 